\title{
Cucurbitacin I induces apoptosis in ovarian cancer cells through oxidative stress and the p190B-Rac1 signaling axis
}

\author{
RULI LI ${ }^{1,2}$, JIANBO XIAO ${ }^{3}$, SUFAN TANG $^{4}$, XINJING LIN $^{4}$, \\ HONGLIN XU ${ }^{4}$, BIN HAN $^{1}$, MING YANG ${ }^{1}$ And FU LIU ${ }^{1}$ \\ ${ }^{1}$ Department of Pharmacy, Affiliated Hospital of North Sichuan Medical College; \\ ${ }^{2}$ Translational Medicine Research Center; ${ }^{3}$ Department of Medical Imaging; ${ }^{4}$ School of Pharmacy, \\ North Sichuan Medical College, Nanchong, Sichuan 637000, P.R. China
}

Received January 10, 2020; Accepted June 12, 2020

DOI: $10.3892 / \mathrm{mmr} .2020 .11327$

\begin{abstract}
Ovarian cancer is a serious threat to women's life and health, with a high mortality rate. Therefore, in addition to improving surgery for ovarian cancer, it is particularly important to develop novel drug treatments. In the present study, the anticancer effects of cucurbitacin I, a natural product, were investigated. Cucurbitacin I impaired the viability of SKVO3 cells in a concentration- and time-dependent manner. Apoptosis was involved in the process of cucurbitacin I-induced cell death, with an increase observed in cleaved-caspase 3 and BAX, and a decrease in Bcl-2. Cucurbitacin I caused a notable increase in intracellular reactive oxygen species, and regulated Kelch-like ECH-associated protein 1 and nuclear factor erythroid-derived 2-like 2 to decrease the expression of antioxidant-related genes. In addition, Cucurbitacin I induced cell shrinkage by regulating the p190BRhoGAP (p190B)-Rac1 signaling axis related to the cytoskeleton. In brief, these results suggested that cucurbitacin I induced cell death through oxidative stress and the p190B-Rac1 signaling axis in SKVO3 cells. The results may provide novel evidence for the treatment of ovarian cancer.
\end{abstract}

\section{Introduction}

Ovarian cancer is one of the most lethal types of cancers in women worldwide. It is the fifth leading cause of cancer-related deaths among women in the United States and causes $>140,000$ deaths annually in women worldwide (1). The pathogenesis of ovarian cancer is very complicated, making it difficult to diagnose and treat (2). Despite continuous improvements in

Correspondence to: Professor Fu Liu, Department of Pharmacy, Affiliated Hospital of North Sichuan Medical College, North Sichuan Medical College, 63 Wenhau Street, Nanchong, Sichuan 637000, P.R. China

E-mail:nclflf91@163.com

Key words: cucurbitacin I, ovarian cancer, apoptosis, oxidative stress, p190BRhoGAP treatment technology, the 5-year survival rate of ovarian cancer remains unsatisfactory. The standard curative treatment for ovarian cancer is anticancer treatment following surgery $(3,4)$. Therefore, in addition to improving diagnostic technology, it is also important to develop new drugs for the treatment of ovarian cancer.

Cucurbitacin I, a natural tetracyclic triterpenoid extracted from Cucurbitaceae and Cruciferae, has been used in traditional medicine for its antipyretic, analgesic, anti-inflammatory and antimicrobial effects (5). According to recent findings, cucurbitacin I, which is 1 of 12 variants of cucurbitacins, has demonstrated a potent anticancer effect on non-small cell lung cancer cells (6). Cucurbitacin I has been reported to cause cancer cell death by inhibiting multiple signaling pathways, such as the Janus kinase (JAK)/STAT3, PI3K/AKT/p70S6K and serine/threonine-protein kinase PAK1 (PAK1)/PAK4 pathways (7-9). However, the underlying mechanism for the anticancer effect of cucurbitacin I is not yet completely understood, and further studies are required to clarify it.

A low level of reactive oxygen species (ROS) is normal in cells, as it helps maintain cell function, but excessive levels of ROS can damage cells and induce cell death. There are regulatory mechanisms that neutralize excessive ROS and provide protection against oxidative damage (10). The transcription factor nuclear factor erythroid-derived 2-like 2 (Nrf2) is an important regulator of the oxidative stress response, and plays a key role in the expression of antioxidant proteins. Nrf2 is regulated by Kelch-like ECH-associated protein 1 (Keap1). Under quiescent conditions, Nrf2 is retained in the cytoplasm after being combined with Keap1, and is degraded in the proteasome with the E3 ubiquitin ligase Cullin 3. Cellular stimuli, such as oxidative stress, induce conformational changes in Keap1, which are followed by the release of Nrf2 from Keap1. Subsequently, Nrf2 translocates to the nucleus and transactivates the expression of genes containing an antioxidant response element in their promoter regions. Nrf2 thereby upregulates phase II detoxifying enzymes and antioxidant proteins, and plays a vital role in maintaining cellular homeostasis $(11,12)$.

p190BRhoGAP (p190B), which is a GTPase-activating protein, has been implicated in various pathological conditions, including cancer, cardiovascular diseases and developmental 
disorders $(13,14)$. As reported by numerous studies, p190B plays an important role in regulating small GTPase activity, which influence cytoskeleton remodeling, cell adhesion, cell polarity maintenance, proliferation and division, as well as cell migration (14,15). A previous study demonstrated that p190B can regulate Ras homolog family member A (RhoA) and Rac1 to regulate cell migration (16). Meanwhile, p190B is critical in the occurrence and development of tumors, such as breast cancer, glioma and colorectal cancer $(17,18)$.

In the present study, cucurbitacin I, a botanical extract, induced death in SKVO3 cells in a time- and concentration-dependent manner. Cucurbitacin I promoted cell apoptosis and induced an increase in the intracellular ROS level, with decreased antioxidant-related gene expression by the Keap1-Nrf2 signaling axis. The expression of p190B and Racl was also found to be altered, which was associated with changes in the cell morphology. The present study may have provided novel evidence for the treatment of ovarian cancer.

\section{Materials and methods}

Reagents. Cucurbitacin I and dimethyl sulfoxide (DMSO) were purchased from Merck KGaA. Cell Counting Kit-8 (CCK-8) was purchased from Bimake. ROS assay kit was obtained from Beyotime Institute of Biotechnology. Anti-BAX (cat. no. 2772) and anti-cleaved-caspase-3 (cat. no. 9664) were from Cell Signaling Technology, Inc. Anti-Bcl-2 (cat. no. ET1603-11) was from Hangzhou HuaAn Biotechnology Co., Ltd. Anti-Keap1 (cat. no. 10503-2-AP), anti-Nrf2 (cat. no. 16396-1-AP), anti-Heme oxygenase-1 (HO-1; cat. no. 10701-1-AP), anti-p190B (cat. no. 55165-1-AP) and anti-Rac1 (cat. no. 24072-1-AP) were purchased from ProteinTech Group, Inc. Anti-GAPDH (cat. no. GB11002) was from Wuhan Servicebio Technology Co., Ltd., and all secondary antibodies (cat. nos. ZB-2301 and ZB-2305) were from Beijing Zhongshan Golden Bridge Biotechnology Co., Ltd. Other chemicals and reagents were analytically pure.

Cell culture. SKOV3 human ovarian carcinoma cells (ATCC HTB-77) were obtained from American Type Culture Collection. SKVO3 cells (70-80\%) were cultured in Dulbecco's modified Eagle's medium (DMEM; HyClone; GE Healthcare) supplemented with $10 \%$ fetal bovine serum (FBS; Gibco; Thermo Fisher Scientific, Inc.), $100 \mathrm{mg} / \mathrm{ml}$ streptomycin and $100 \mathrm{U} / \mathrm{ml}$ penicillin. SKVO3 cells were incubated in a humidified incubator containing $95 \%$ air and $5 \% \mathrm{CO}_{2}$ with the temperature stabilized at $37^{\circ} \mathrm{C}$. The cell culture media was refreshed every 3 days.

Cell viability assays. Cell viability assays were performed according to a previous study (19). SKVO3 cells were seeded into 96-well plates at a density of 10,000 cell per well. The experimental groups were treated with $0.075,0.15,0.3$ and $0.6 \mu \mathrm{M}$ cucurbitacin I (dissolved in DMSO) for 12, 24 and $48 \mathrm{~h}$, and the control group was treated with DMSO. Finally, a CCK-8 assay was performed, according to the manufacturer's protocol, to detect cell viability using a microplate reader (BioTek). As $0.3 \mu \mathrm{M}$ cucurbitacin I treatment for $24 \mathrm{~h}$ could effectively reduce cell viability by $\sim 50 \%$, this concentration and time was chosen for subsequent experiments.
ROS assay. The ROS assay was performed according to a previous study (20). ROS were measured using a fluorescent probe DCFH-DA (Beyotime Institute of Biotechnology). SKVO3 cells $\left(2 \times 10^{5}\right.$ per well) were seeded into 6 -well plates, and then when cells reached $\sim 80 \%$ confluence they were treated with $0.3 \mu \mathrm{M}$ cucurbitacin I for $24 \mathrm{~h}$ at $37^{\circ} \mathrm{C}$ (control cells were treated with DMSO). All cells were further incubated with $10 \mu \mathrm{M}$ DCFH-DA for $20 \mathrm{~min}$, and then washed with serum-free DMEM 3 times. The level of ROS was detected using a fluorescence microscope.

Western blot analysis. Western blotting was performed as previously described $(21,22)$. SKVO3 cells were lysed on ice for $20 \mathrm{~min}$, centrifuged at $10,000 \mathrm{x}$ for $10 \mathrm{~min}$ at $4^{\circ} \mathrm{C}$ and the cell supernatant was collected. Protein concentration was measured by the BCA method, and $35 \mu \mathrm{g}$ protein per lane was loaded onto 10 or $12 \%$ gels and separated via SDS-PAGE. The separated proteins were subsequently transferred onto polyvinylidene fluoride membranes. The membranes were incubated with $5 \%$ skimmed milk diluted by TBS with $0.1 \%$ Tween-20 (TBST) for $1 \mathrm{~h}$ at room temperature, and then probed with primary antibodies $(1: 1,000)$ at $4^{\circ} \mathrm{C}$ overnight. The membranes were washed with TBST 3 times and incubated with the secondary antibodies $(1: 3,000)$ for $1 \mathrm{~h}$ at room temperature to amplify the chemiluminescence. Bands were semi-quantified and analyzed using ImageJ Software (V1.8.0.112; National Institutes of Health).

Statistical analysis. All data are expressed as the mean \pm SEM. Comparisons were performed using an unpaired Student's t-test and groups of $\geq 3$ were analyzed by one-way ANOVA, followed by Tukey's post hoc tests. $\mathrm{P}<0.05$ was considered to indicate a statistically significant difference.

\section{Results}

Cucurbitacin I causes SKVO3 cell death in a concentrationand time-dependent manner. To evaluate the cytotoxicity of cucurbitacin I on SKVO3 cells, the cell viability of SKVO3 was detected by a CCK-8 assay. As shown in Fig. 1A, cucurbitacin I treatment induced cell death in a concentration- and time-dependent manner. Briefly, the cucurbitacin I concentration used was $0.075-0.6 \mu \mathrm{M}$. The higher the concentration, the higher the death rate and the lower the survival rate. Cells were treated with cucurbitacin I for 12, 24 and $48 \mathrm{~h}$. The longer the treatment time, the higher the cell death rate and the lower the survival rate. Cell death is always accompanied by changes in cell morphology. As shown in Fig. 1B, following cucurbitacin I treatment for $24 \mathrm{~h}$, there was a decreased number of SKVO3 cells and cell surface shrinkage; the higher the concentration, the more severe this was.

Cucurbitacin I induces apoptosis in SKVO3 cells. To determine whether cucurbitacin I induced cell apoptosis, a concentration of $0.3 \mu \mathrm{M}$ was selected as it was shown to kill $\sim 50 \%$ of SKVO3 cells. It was observed that treatment with $0.3 \mu \mathrm{M}$ cucurbitacin I for $24 \mathrm{~h}$ induced a significant decrease in $\mathrm{Bcl}-2(37.3 \%, \mathrm{P}<0.05)$ and a significant increase in BAX $(80.2 \%, \mathrm{P}<0.05)$. The expression level of cleaved-caspase- 3 , an index of apoptosis, was also examined, and it was found to 
A

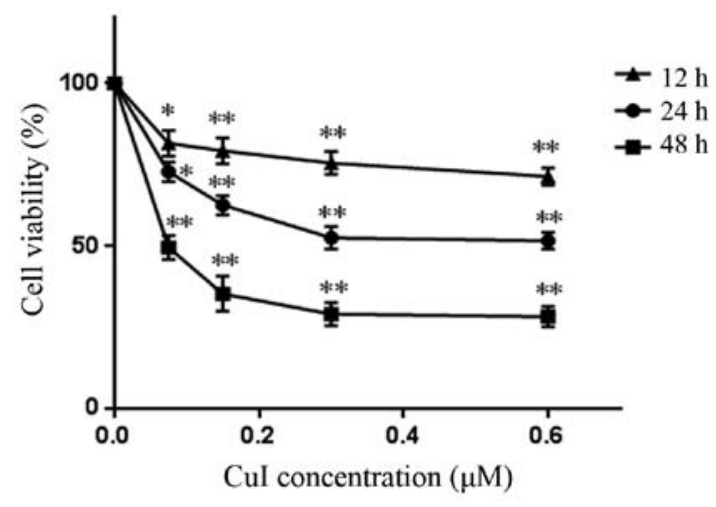

B

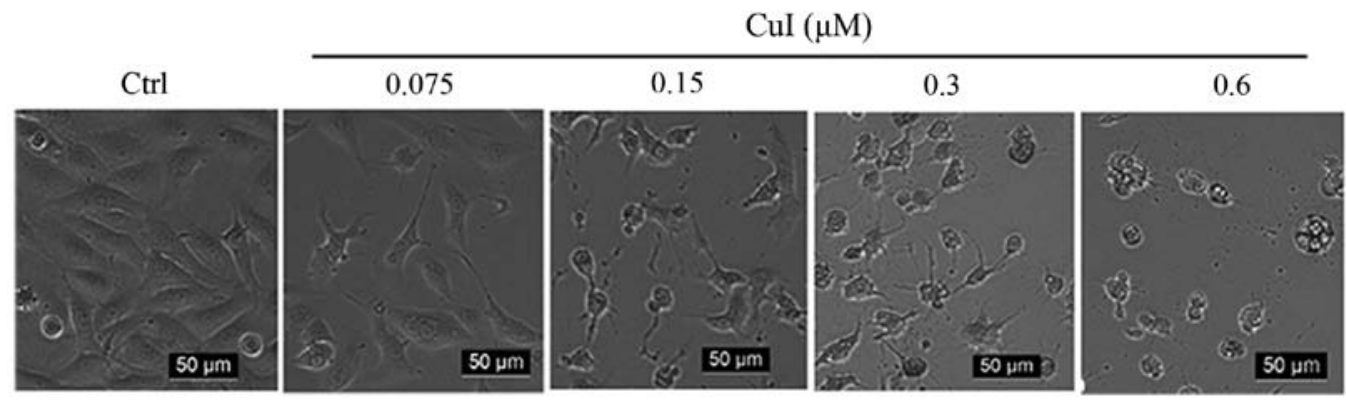

Figure 1. CuI causes SKVO3 cell death in a concentration- and time-dependent manner. (A) SKVO3 cells were treated with $0.075,0.15,0.3$ and $0.6 \mu \mathrm{M} \mathrm{CuI} \mathrm{for}$ 12,24 and $48 \mathrm{~h}$, whereas the control group was treated with DMSO alone. Cell viability was then measured by Cell Counting Kit- 8 assay ( $\mathrm{n}=3 / \mathrm{group}$ ). (B) Cells were visualized using an inverted microscope. Scale bars, $50 \mu \mathrm{m}$; magnification, $\mathrm{x} 200$. Data were analyzed with one-way ANOVA followed by Tukey's post hoc test and are presented as the mean \pm SEM. ${ }^{*} \mathrm{P}<0.05,{ }^{* *} \mathrm{P}<0.01$ vs. DMSO-treated group (control). Ctrl, DMSO-treated cells; DMSO, dimethyl sulfoxide; CuI, cucurbitacin I.

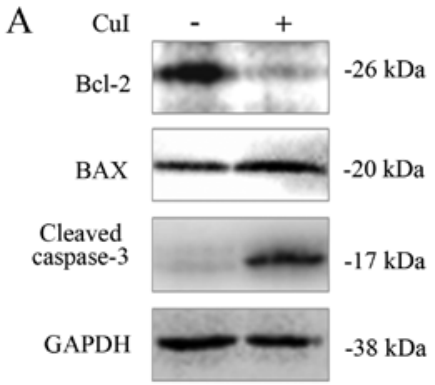

$\mathrm{C}$

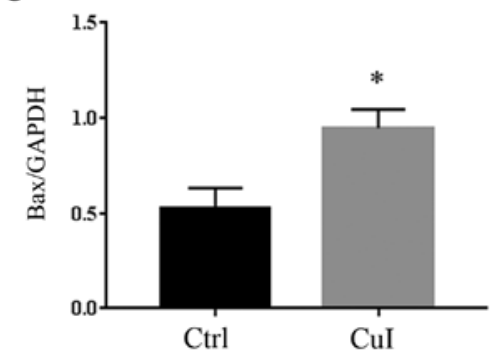

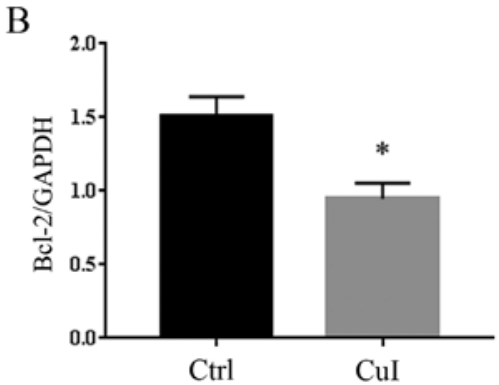

D

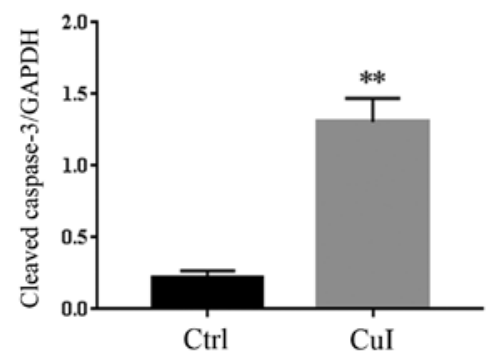

Figure 2. CuI induces apoptosis in SKVO3 cells. (A) Representative protein expression of apoptosis markers, Bcl-2, BAX and cleaved-caspase-3, in SKVO3 cells treated with $0.3 \mu \mathrm{M} \mathrm{CuI}$ for $24 \mathrm{~h}$. The control group was treated with DMSO alone. (B-D) Corresponding histograms of the protein expression of apoptosis markers in CuI-treated SKVO3 cells ( $\mathrm{n}=3$ /group). All data were analyzed with an unpaired Student's t-test and are presented as the mean $\pm \mathrm{SEM}$. " $\mathrm{P}<0.05$, ${ }^{* *} \mathrm{P}<0.01$ vs. Ctrl. Ctrl, control; CuI, cucurbitacin I.

have increased by $380 \%(\mathrm{P}<0.01)$ compared with the control group treated with DMSO alone (Fig. 2).

Cucurbitacin I increases cellular oxidative stress via the Keapl-Nrf2 pathway. To evaluate the effect of cucurbitacin I on oxidative stress, the level of ROS in SKVO3 cells was measured. As shown in Fig. 3A, when cells were treated with $0.3 \mu \mathrm{M}$ cucurbitacin I, the level of intracellular ROS markedly increased, whereas that of the control group was very low. Cells have protective mechanisms against oxidative stress, including a 
A

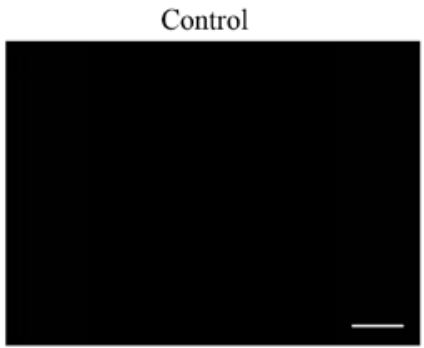

B

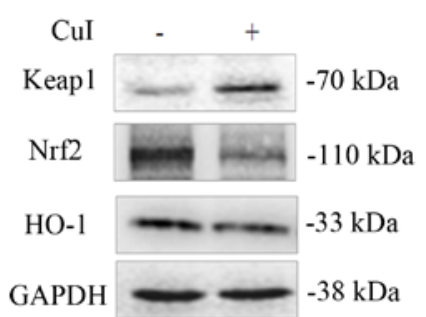

D

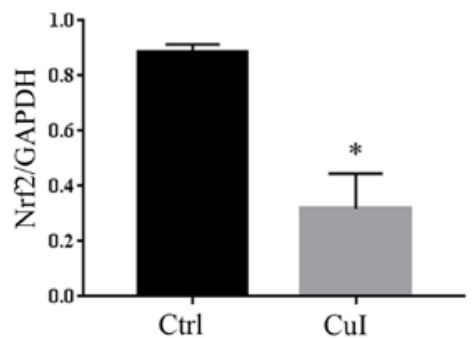

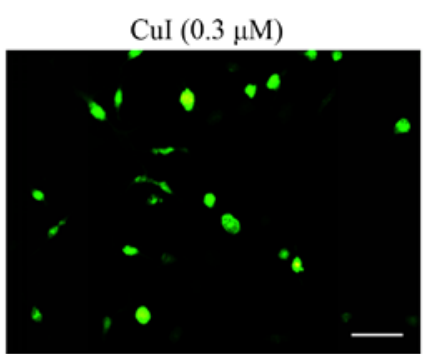

$\mathrm{C}$

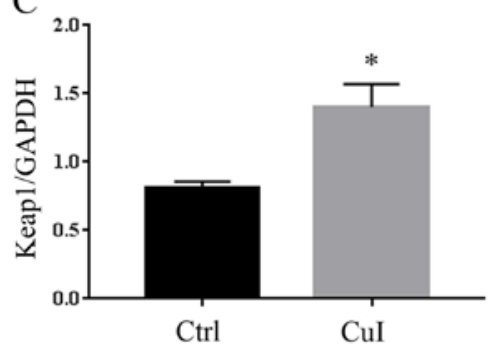

E

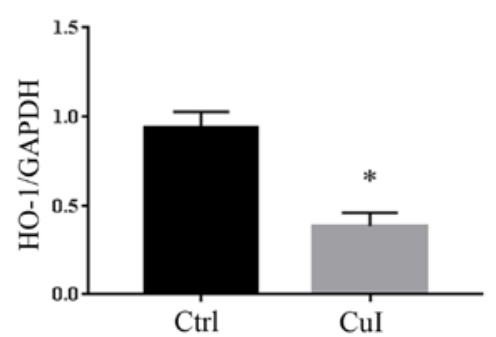

Figure 3. CuI increases cellular oxidative stress via the Keap1-Nrf2 pathway. (A) Representative fluorescence images of ROS in SKVO3 cells were captured using a fluorescent microscope with DCFH-DA stain. Scale bar, $50 \mu \mathrm{m}$. (B) Representative protein expression of Keap1, Nrf2 and HO-1 in SKVO3 cells. (C-E) Corresponding histograms of the protein expression of Keap1, Nrf2 and HO-1 (n=3/group). All data were analyzed with an unpaired Student's t-test and are presented as the mean \pm SEM. * $<<0.05$ vs. Ctrl. Ctrl, control; Keap1, Kelch-like ECH-associated protein 1; Nrf2, nuclear factor erythroid-derived 2-like 2; ROS, reactive oxygen species; HO-1, Heme oxygenase-1; CuI, cucurbitacin I.

number of antioxidant proteins and signaling pathways, of which the Keap1-Nrf2 pathway is one of them. Therefore in the present study, the expression of Keap1, Nrf2 and HO-1, which is downstream of Nrf2, was measured. As shown in Fig. 3B-E, the protein expression of Nrf2 decreased by $63.9 \%(\mathrm{P}<0.05)$, HO-1 decreased by $58.7 \%(\mathrm{P}<0.05)$ and Keap1 increased by $72.5 \%(\mathrm{P}<0.05)$ compared with the control group. These results indicated that that cucurbitacin I increased the level of intracellular oxidative stress and therefore regulated the Keap1-Nrf2 pathway.

Cucurbitacin I alters cell morphology by regulating the p190B-Racl pathway. It has been reported that the expression of p190B is increased in ovarian cancer, which may affect the cell cytoskeleton, morphology and migration (23). As shown in Fig. 1B, the number of cells decreased and the cell surface had shrunk with the increase in cucurbitacin I concentration. Therefore, the expression levels of cytoskeleton-related protein p190B and its downstream protein Rac1 were detected. The results showed that, as compared with the control group, the expression of $190 \mathrm{~B}$ decreased by $42.8 \%(\mathrm{P}<0.01)$ and $\mathrm{Racl}$ expression increased by $66.3 \%(\mathrm{P}<0.05)$ in cells treated with $0.3 \mu \mathrm{M}$ cucurbitacin I for $24 \mathrm{~h}$ (Fig. 4). Thus, suggesting that cucurbitacin I regulated the p190B and Rac1 pathway to regulate cell morphology.

\section{Discussion}

Ovarian cancer is one of the most common malignant tumors in female reproductive organs. The incidence of ovarian cancer is second only to that of cervical cancer and uterine body cancer, and is a serious threat to women's lives (2-4). The etiology of ovarian cancer is still unclear, but may be related to genetic and endocrine factors. At present, in addition to surgery, the development of antitumor drugs is also very important. Cucurbitacin I has a strong antitumor activity (6,7). Cucurbitacin I has been reported to induce death in various types of tumor cells; for example it can induce apoptosis in non-small cell lung cancer cells and osteosarcoma cells by inhibiting the PI3K/AKT/p70S6K and STAT3 signaling, respectively. Zhang et al (24) observed that cucurbitacin I modulated the balance between autophagic and apoptotic modes of cells to cause cancer cell death. In the present study, the SKVO3 cell line was selected to investigate ovarian cancer via various experiments. The present findings confirmed that cucurbitacin I could kill SKVO2 cells in a concentration- and time-dependent manner, indicating that its antitumor effect was enhanced as the concentration increased. As shown in Fig. 1, a noteworthy phenomenon was found, after cucurbitacin I administration, some of the shrunk cells that had attached to the culture dish still had cell viability and did not 
A

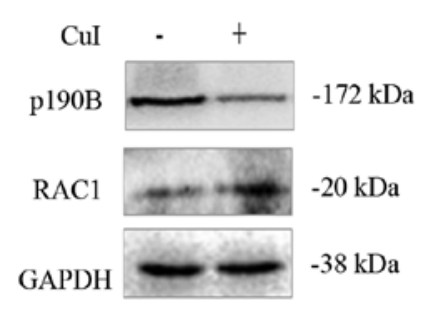

B

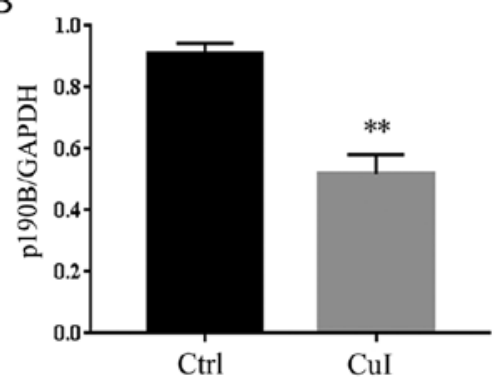

C

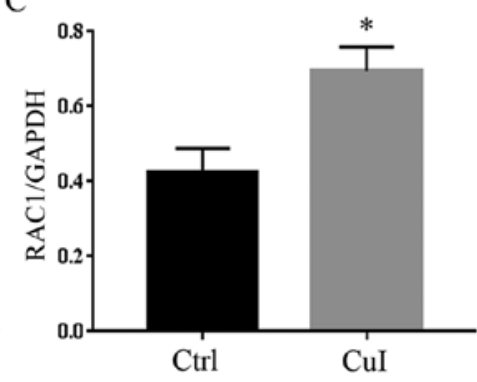

Figure 4. CuI regulates the p190B-Rac1 pathway. (A) Representative protein expression of p190B and Rac1 in SKVO3 cells. (B and C) Corresponding histograms of the protein expression of $\mathrm{p} 190 \mathrm{~B}$ and Racl ( $\mathrm{n}=3$ /group). All data were analyzed using an unpaired Student's $\mathrm{t}$-test and are presented as the mean \pm SEM. ${ }^{*} \mathrm{P}<0.05,{ }^{* *} \mathrm{P}<0.01$ vs. Ctrl. Ctrl, control; CuI, cucurbitacin I; p190B, p190BRhoGAP.

die completely. The morphology of SKVO3 cells changed in a concentration-dependent manner.

Apoptosis is a form of programmed cell death that brings about the orderly and efficient removal of damaged cells, such as those resulting from DNA damage or during development $(25,26)$. The mechanism of apoptosis is very complex and numerous signaling pathways are involved in this process. However, caspases, a family of cysteinyl-aspartate-specific proteases, are central to the mechanism of apoptosis $(27,28)$. The activity of caspases is responsible for the hallmarks of apoptosis, so the expression level of cleaved-caspase-3 was detected. As shown in Fig. 2A, cucurbitacin I caused a significant increase in the expression level of cleaved-caspase-3 and pro-apoptotic protein BAX in SKVO3 cells, whereas the expression of anti-apoptotic protein Bcl-2 decreased and the ratio of Bcl-2/BAX decreased. In general, these results indicated that cucurbitacin I induced SKVO3 cell apoptosis.

Cancer development is characterized by the uncontrolled growth and proliferation of transformed cells. A tumor environment is characterized by low levels of oxygen and glucose, which can induce ROS. At moderate concentrations, ROS activates the cancer cell survival signaling cascade. At high concentrations, ROS can cause cancer cell apoptosis (29). In the present study, the mechanism through which cucurbitacin I induced cell death in ovarian cancer was further explored. It was found that cucurbitacin I increased the level of ROS in cells and decreased the expression of antioxidant genes. Briefly, cucurbitacin I decreased the expression of Nrf2, but increased that of the Keap1 protein, which can combine with Nrf2 to further prevent Nrf2 from translocating into the nucleus. Next, the expression of HO-1, an antioxidant gene downstream of $\mathrm{Nrf} 2$, was detected, and it was found to have decreased by $58.7 \%$. Generally speaking, cucurbitacin I increased the level of ROS and decreased the expression of antioxidant genes via the Keap1-Nrf2 signaling axis, resulting in cell death.

When ovarian cancer occurs, the expression of cytoskeleton-related proteins increases. Earp et al (23) suggested that the expression of the p190B protein increased significantly during the development of ovarian cancer. Therefore, reducing the expression of p190B could be a potential target for treatment. In the present study, following the treatment of SKVO3 cells by cucurbitacin I, the expression of $\mathrm{p} 190 \mathrm{~B}$, a regulatory protein associated with the cytoskeleton, decreased significantly, and that of the negative regulatory protein Racl increased. This was associated with the morphological changes of cells in Fig. 1B.
The anti-tumor signaling pathway of cucurbitacin $\mathrm{I}$ is very complex. Although, it has been reported by Li et al (30) that cucurbitacin I causes ovarian cancer cell death via endoplasmic reticulum stress, the present study demonstrated for the first time, to the best of the our knowledge, that cucurbitacin I can also activate oxidative stress by regulating the Keap1-Nrf2-HO1 signaling pathway, and regulate the p190b-Rac1 signal axis to alter cell cytoskeleton shape, so as to achieve the anti-tumor effect. A limitation of the present study is that only one cell line was used and so there was a lack of dose responses in this experiment, in future experiments other cell lines, as well as primary cells, will be used to confirm our findings. Additionally, further experiments to determine concentration gradient are needed, and a variety of research methods, such as flow cytometry and TUNEL fluorescence detection, will be performed to further explore the findings of the present study.

In conclusion, cucurbitacin I, a newly identified potential chemotherapeutic drug, induced ovarian cancer cell death in a concentration- and time-dependent manner. Cucurbitacin I induced cell apoptosis by increasing BAX and cleaved-caspase-3, and decreasing Bcl-2. In addition, cucurbitacin I increased the intracellular ROS level and decreased antioxidant gene expression via the Keap1-Nrf2 signaling pathway. In addition, the p190B-Rac1 signaling axis was inhibited by cucurbitacin I. These insights may be used to develop novel therapeutic approaches to treat ovarian cancer.

\section{Acknowledgements}

Not applicable.

\section{Funding}

The present study was supported by the National Natural Science Foundation of China (grant no. 81900276), Nanchong City \& School Cooperation Project (grant no. 18SXHZ0546) and Sichuan Provincial Health Commission's popularization and application project(grant no. 20PJ146)

\section{Availability of data and materials}

The datasets used and/or analyzed during the current study are available from the corresponding author on reasonable request. 


\section{Authors' contributions}

RL and FL performed the experiments and drafted the manuscript. JX, XL, HX and ST participated in the experiments. MY and BH contributed to the final data analysis and edited the manuscript. All the authors read and approved the final manuscript.

\section{Ethics approval and consent to participate}

Not applicable.

\section{Patient consent for publication}

Not applicable.

\section{Competing interests}

The authors declare that they have no competing interests.

\section{References}

1. Hunn J and Rodriguez GC: Ovarian cancer: Etiology, risk factors, and epidemiology. Clin Obstet Gynecol 55: 3-23, 2012.

2. Farsinejad S, Cattabiani T, Muranen T and Iwanicki M: Ovarian cancer dissemination-A cell Biologist's perspective. Cancers (Basel) 11: 1957, 2019.

3. Yeung TL, Leung CS, Yip KP, Au Yeung CL, Wong ST and Mok SC: Cellular and molecular processes in ovarian cancer metastasis. A review in the theme: Cell and molecular processes in cancer metastasis. Am J Physiol Cell Physiol 309: C444-C456, 2015.

4. Moufarrij S, Dandapani M, Arthofer E, Gomez S, Srivastava A, Lopez-Acevedo M, Villagra A and Chiappinelli KB: Epigenetic therapy for ovarian cancer: Promise and progress. Clin Epigenetics 11: 7, 2019.

5. Xu K, Xiao J, Zheng K, Feng X, Zhang J, Song D, Wang C, Shen X, Zhao X, Wei C, et al: miR-21/STAT3 signal is involved in odontoblast differentiation of human dental pulp stem cells mediated by TNF- $\alpha$. Cell Reprogram 20: 107-116, 2018.

6. Ni Y, Wu S, Wang X, Zhu G, Chen X, Ding Y and Jiang W: Cucurbitacin I induces pro-death autophagy in A549 cells via the ERK-mTOR-STAT3 signaling pathway. J Cell Biochem 119: 6104-6112, 2018.

7. Zhu X, Huang H, Zhang J, Liu H, Ao R, Xiao M and Wu Y: The anticancer effects of Cucurbitacin I inhibited cell growth of human non-small cell lung cancer through PI3K/AKT/p70S6K pathway. Mol Med Rep 17: 2750-2756, 2018.

8. Yang Q, Qiu H, Xie H, Qi Y, Cha H, Qu J, Wang M, Feng Y, Ye X, Mu J and Huang J: A Schistosoma japonicum infection promotes the expansion of Myeloid-derived suppressor cells by activating the JAK/STAT3 pathway. J Immunol 198: 4716-4727, 2017.

9. Nguyen BC, Taira N, Maruta H and Tawata S: Artepillin C and other Herbal PAK1-blockers: Effects on hair cell proliferation and related PAK1-dependent biological function in cell culture. Phytother Res 30: 120-127, 2016.

10. Moloney JN and Cotter TG: ROS signalling in the biology of cancer. Semin Cell Dev Biol 80: 50-64, 2018

11. Zhang Y, Yan T, Sun D, Xie C, Wang T, Liu X, Wang J, Wang Q, Luo Y, Wang P, et al: Rutaecarpine inhibits KEAP1-NRF2 interaction to activate NRF2 and ameliorate dextran sulfate sodium-induced colitis. Free Radic Biol Med 148: 33-41, 2020.

12. Wang J, Zhang W, Lv C, Wang Y, Ma B, Zhang H, Fan Z, Li M and Li X: A novel biscoumarin compound ameliorates cerebral ischemia reperfusion-induced mitochondrial oxidative injury via Nrf2/Keap-1/ARE signaling. Neuropharmacology 167: 107918, 2020 .
13. Tian T, Chen ZH, Zheng Z, Liu Y, Zhao Q, Liu Y, Qiu H, Long Q, Chen M, Li L, et al: Investigation of the role and mechanism of ARHGAP5-mediated colorectal cancer metastasis. Theranostics 10: 5998-6010, 2020.

14. Héraud C, Pinault M, Lagrée V and Moreau V: p190RhoGAPs, the ARHGAP35- and ARHGAP5-encoded proteins, in health and disease. Cells 8: 351, 2019.

15. Frank SR, Köllmann CP, Luong P, Galli GG, Zou L, Bernards A Getz G, Calogero RA, Frödin M and Hansen SH: p190 RhoGAP promotes contact inhibition in epithelial cells by repressing YAP activity. J Cell Biol 217: 3183-3201, 2018.

16. Bustos RI, Forget MA, Settleman JE and Hansen SH: Coordination of Rho and Rac GTPase function via p190B RhoGAP. Curr Biol 18: 1606-1611, 2008.

17. Hwang M, Peddibhotla S, McHenry P, Chang P, Yochum Z, Park KU, Sears JC and Vargo-Gogola T: P190B RhoGAP regulates chromosome segregation in cancer cells. Cancers (Basel) 4: 475-489, 2012.

18. Heckman-Stoddard BM, Vargo-Gogola T, McHenry PR, Jiang V, Herrick MP, Hilsenbeck SG, Settleman J and Rosen JM: Haploinsufficiency for p190B RhoGAP inhibits MMTV-Neu tumor progression. Breast Cancer Res 11: R61, 2009.

19. Zhai KF, Duan H, Cui CY, Cao YY, Si JL, Yang HJ, Wang YC, Cao WG, Gao GZ and Wei ZJ: Liquiritin from Glycyrrhiza uralensis attenuating rheumatoid arthritis via reducing inflammation, suppressing angiogenesis, and inhibiting MAPK signaling pathway. J Agric Food Chem 67: 2856-2864, 2019.

20. Wang H and Joseph JA: Quantifying cellular oxidative stress by dichlorofluorescein assay using microplate reader. Free Radic Biol Med 27: 612-616, 1999.

21. Zhai KF, Zheng JR, Tang YM, Li F, Lv YN, Zhang YY, Gao Z, Qi J, Yu BY and Kou JP: The Saponin D39 blocks dissociation of non-muscular myosin heavy chain IIA from TNF receptor 2 , suppressing tissue factor expression and venous thrombosis. $\mathrm{Br}$ J Pharmacol 174: 2818-2831, 2017.

22. Li RL, Wu SS, Wu Y, Wang XX, Chen HY, Xin JJ, Li H, Lan J, Xue KY, Li X, et al: Irisin alleviates pressure overload-induced cardiac hypertrophy by inducing protective autophagy via mTOR-independent activation of the AMPK-ULK1 pathway. J Mol Cell Cardiol 121: 242-255, 2018.

23. Earp M, Tyrer JP, Winham SJ, Lin HY, Chornokur G, Dennis J, Aben KKH, Anton-Culver H, Antonenkova N, Bandera EV, et al: Variants in genes encoding small GTPases and association with epithelial ovarian cancer susceptibility. PLoS One 13: e0197561, 2018.

24. Zhang T, Li Y, Park KA, Byun HS, Won M, Jeon J, Lee Y, Seok JH, Choi SW, Lee SH, et al: Cucurbitacin induces autophagy through mitochondrial ROS production which counteracts to limit caspase-dependent apoptosis. Autophagy 8: 559-576, 2012.

25. Dietz A, Dalda N, Zielke S, Dittmann J, van Wijk SJL, Vogler M and Fulda S: Proteasome inhibitors and Smac mimetics cooperate to induce cell death in diffuse large B-cell Lymphoma by stabilizing NOXA and triggering mitochondrial apoptosis. Int J Cancer 4: 982, 2020.

26. Malinina A, Dikeman D, Westbrook R, Moats M, Gidner S, Poonyagariyagorn H, Walston J and Neptune ER: IL10 deficiency promotes alveolar enlargement and lymphoid dysmorphogenesis in the aged murine lung. Aging Cell 19: e13130, 2020.

27. Zhai KF, Duan H, Chen Y, Khan GJ, Cao WG, Gao GZ, Shan LL and Wei ZJ: Apoptosis effects of imperatorin on synoviocytes in rheumatoid arthritis through mitochondrial/caspase-mediated pathways. Food Funct 9: 2070-2079, 2018.

28. Ma M, Wang X, Liu N, Shan F and Feng Y: Low-dose naltrexone inhibits colorectal cancer progression and promotes apoptosis by increasing M1-type macrophages and activating the $\mathrm{Bax} / \mathrm{Bcl}-2 /$ caspase-3/PARP pathway. Int Immunopharmacol 83: $106388,2020$.

29. Aggarwal V, Tuli HS, Varol A, Thakral F, Yerer MB, Sak K, Varol M, Jain A, Khan MA and Sethi G: Role of reactive oxygen species in cancer progression: Molecular mechanisms and recent advancements. Biomolecules 9: 735, 2019.

30. Li H, Chen H, Li R, Xin J, Wu S, Lan J, Xue K, Li X, Zuo C, Jiang $\mathrm{W}$ and Zhu L: Cucurbitacin I induces cancer cell death through the endoplasmic reticulum stress pathway. J Cell Biochem: Sep 11, 2018 (Epub ahead of print). 